\title{
Role for central ghrelin in food intake and secretion profile of stomach ghrelin in rats
}

\author{
N Murakami, T Hayashida, T Kuroiwa, K Nakahara, T Ida, \\ M S Mondal', M Nakazato', M Kojima² and K Kangawa ${ }^{3}$ \\ Department of Veterinary Physiology, Faculty of Agriculture, University of Miyazaki, Miyazaki 889-2155, Japan \\ ${ }^{1}$ Third Department of Internal Medicine, Miyazaki Medical College, Kiyotake, Miyazaki 889-1692, Japan \\ ${ }^{2}$ Molecular Genetics, Institute of Life Science, Kurume-University, Kurume, Japan \\ ${ }^{3}$ National Cardiovascular Center Research Institute, Osaka 565-8565, Japan \\ (Requests for offprints should be addressed to N Murakami; Email: a0d201u@cc.miyazaki-u.ac.jp)
}

\begin{abstract}
Ghrelin, a 28-amino-acid peptide, has recently been isolated from the rat stomach as an endogenous ligand for the GH secretagogue receptor. We have reported previously that central or peripheral administration of ghrelin stimulates food intake, and the secretion of $\mathrm{GH}$ and gastric acid in rats. In the present study, we investigated how much endogenous centrally released ghrelin is involved in the control of food intake and body weight gain. We also examined the profile of ghrelin secretion from the stomach by RIA using two kinds of anti-ghrelin antiserum, one raised against the $\mathrm{N}$-terminal $\left(\left[\mathrm{Cys}^{12}\right]\right.$-ghrelin[1-11]) region and one raised against the $\mathrm{C}$-terminal $\left(\left[\mathrm{Cys}^{0}\right]\right.$-ghrelin [13-28]) region of the peptide. The former antibody recognizes specifically ghrelin with $n$-octanoylated Ser 3 (acyl ghrelin), and does not recognize des-acyl ghrelin. The latter also recognizes des-acyl ghrelin (i.e. total ghrelin). Intracerebroventricular treatment with the antighrelin antiserum against the $\mathrm{N}$-terminal region twice a
\end{abstract}

day for 5 days decreased significantly both daily food intake and body weight. Des-acyl ghrelin levels were significantly higher in the gastric vein than in the trunk. Either fasting for $12 \mathrm{~h}$, administration of gastrin or cholecystokinin resulted in increase of both acyl and des-acyl ghrelin levels. The ghrelin levels exhibited a diurnal pattern, with the bimodal peaks occurring before dark and light periods. These two peaks were consistent with maximum and minimum volumes of gastric content respectively. These results suggest that (1) endogenous centrally released ghrelin participates in the regulation of food intake and body weight, (2) acyl ghrelin is secreted from the stomach, (3) intestinal hormones stimulate ghrelin release from the stomach, and (4) regulation of the diurnal rhythm of ghrelin is complex, since ghrelin secretion is augmented under conditions of both gastric emptying and filling.

Journal of Endocrinology (2002) 174, 283-288

\section{Introduction}

The peptide ghrelin has recently been purified from rat stomach, and has been identified as an endogenous ligand for the growth hormone $(\mathrm{GH})$ secretagogue receptor (GHS-R) (Kojima et al. 1999). Ghrelin consists of 28 amino acids, including an $\mathrm{O}-n$-octanoylated Ser-3 residue, which is essential for GH release. An analogue of ghrelin, des-Gln14-ghrelin, has also been purified from rat stomach, and is a second endogenous ligand for GHS-R (Hosoda et al. 2000). Immunohistochemical studies have revealed that ghrelin is also found in the hypothalamic arcuate nucleus of the brain (Kojima et al. 1999). It has been demonstrated that intracerebroventricular (i.c.v.) or intravenous administration of ghrelin stimulates the secretion of GH (Kojima et al. 1999, Date et al. 2000b, Wren et al. 2000), insulin, gastrin (Lee et al. 2002) and gastric acid (Date et al. 2001), and increases food intake and body weight gain in rodents (Wren et al. 2000, Asakawa et al. 2001, Nakazato et al. 2001). In addition to a single injection, daily i.c.v. treatments with ghrelin also increase food intake and body weight gain in rats (Tschop et al. 2000). These orexigenic actions of ghrelin are mediated by hypothalamic neuropeptide $\mathrm{Y}$ and agouti-related protein (Asakawa et al. 2001, Kamegai et al. 2001, Nakazato et al. 2001, Shintani et al. 2001). These results suggest that ghrelin plays an important role in energy balance and digestive function. However, it is not clear how much endogenous centrally released ghrelin is involved in the control of food intake or body weight gain. In the study presented here, we examined food intake and body weight gain in rats that had been administered anti-ghrelin antiserum i.c.v. twice a day for 5 days. 
Immunohistochemical studies, including in situ hybridization, have revealed that in the gastrointestinal tract, ghrelin is produced mainly from the neck to the base of the oxyntic glands, especially in the X/A-like cells whose physiological role was previously unknown (Date et al. 2000a). Although it is likely that ghrelin is secreted from the stomach to the gastric vein, no detailed profile has yet been demonstrated. Analysis by reverse-phase HPLC (RP-HPLC) has revealed that both acyl and des-acyl ghrelin exist in the rat gastric fundus at a ratio of $1 \cdot 3: 1$ (des-acyl ghrelin : acyl ghrelin) (Toshinai et al. 2001). However, it is not clear whether they are secreted from the stomach. Furthermore, there is little information concerning the regulation of ghrelin secretion from the stomach. Plasma ghrelin levels, as determined by RIA using a ghrelin antiserum against the C-terminal fragment (residues 13-28), were increased by vagotomy, and treatment with thyroxine $\left(\mathrm{T}_{4}\right)$ and leptin did not influence plasma ghrelin levels (Lee et al. 2002). Although in these cases plasma ghrelin was measured after chronic endocrine manipulation that was performed two or three times per day for 3-4 days, it does appear at least that ghrelin secretion is influenced by some gastrointestinal hormones. In a preliminary experiment, plasma ghrelin levels were measured by RIA using two types of anti-ghrelin antiserum (one raised against the N-terminal region and the other against the C-terminal region). The antibody raised against the $\mathrm{N}$-terminal region recognized acyl ghrelin, but not des-acyl ghrelin; that raised against the C-terminal region recognized both acylated and des-acyl ghrelin. These two antibodies provided values that were quite different. In the present study, we compared the plasma levels of acylated and total ghrelin in the gastric vein and trunk. In addition, we examined the acute effects of administration of gastrin and cholecystokinin (CCK), and electrical stimulation of the vagus nerve on ghrelin secretion from the stomach in rats.

\section{Materials and Methods}

\section{Animals and experimental schedule}

Adult male Wistar rats (Charles River Japan Inc., Shiga, Japan) were kept under a light regimen of $12 \mathrm{~h}$ light : $12 \mathrm{~h}$ darkness (light on at $0700 \mathrm{~h}$ ) and a temperature of $23 \pm 1{ }^{\circ} \mathrm{C}$. Animals were supplied with standard laboratory chow and water and allowed to feed ad libitum. All procedures were performed in accordance with the Japanese Physiological Society's guidelines for animal care. All experiments were repeated twice.

In the first experiment, we used 15-week-old rats weighing about $400 \mathrm{~g}$. The body weight at this age has almost reached maximum. Each of four rats were administered a $10 \mu \mathrm{l}$ saline solution of purified anti-ghrelin $\operatorname{IgG}$ $(0.1$ and $1.0 \mu \mathrm{g})$ or $1.0 \mu \mathrm{g}$ pre-immune serum IgG twice a day (at 0845 and $1845 \mathrm{~h}$ ) for 5 days. The anti-ghrelin antiserum used in this study was the type raised against the $\mathrm{N}$-terminal region of ghrelin ( $n$-octanoylated rat [1-11] ghrelin). Food intake and body weight were measured every morning for 5 days. The method of preparation of anti-ghrelin IgG is described elsewhere (Nakazato et al. 2001).

In a second experiment, each of four adult male rats weighing about $300 \mathrm{~g}$ were used for measurements of plasma ghrelin levels in the gastric vein and trunk. Rats were anesthetized with pentobarbital at $1400 \mathrm{~h}$, after which the blood was collected from the gastric vein (Toshinai et al. 2001), and then from the heart puncture. Each $1 \mathrm{ml}$ of truncal or gastric vein blood was drawn into chilled polypropylene tubes containing disodium EDTA (EDTA-2Na; $1 \mathrm{mg} / \mathrm{ml}$ blood), aprotinin (500 units/ml blood) and Pefabloc SC (Roche, Nutley, NJ, USA; $2.4 \mathrm{mg} / \mathrm{ml}$ blood), and then centrifuged. The plasma was stored at $-80^{\circ} \mathrm{C}$ until extraction of ghrelin.

In a third experiment, blood was collected from the gastric vein of each of four rats after $12 \mathrm{~h}$ of fasting (from 1900 to $0700 \mathrm{~h}$ ), and $40 \mathrm{~min}$ after an i.p. injection of either CCK ( $20 \mu \mathrm{g} / \mathrm{kg}$ body weight; Sigma Chemical Co., St Louis, MO, USA), gastrin $(2 \mu \mathrm{g} / \mathrm{kg}$ body weight, Sigma) or saline. In addition, eight rats were anesthetized using pentobarbital, and the left cervical vagus nerve was stimulated electrically for $10 \mathrm{~min}$. This electrical stimulation was performed using bipolar electrodes, with stimulation at a frequency, intensity and duration of $20 \mathrm{~Hz}, 10 \mathrm{~V}$ and $1 \mathrm{~ms}$ respectively, each for $30 \mathrm{~s}$, and at 30-s intervals. It has been shown that this manipulation increases gastric acid secretion by over fivefold compared with the notreatment condition. In four of the eight rats, blood samples were collected from the gastric vein 30 and 90 min after stimulation.

In a fourth experiment, to examine the diurnal pattern of plasma ghrelin levels, three rats were killed by decapitation at 3-h intervals for $24 \mathrm{~h}$. The stomach contents were weighed. A sample of blood was taken for glucose measurement using a commercial assay kit (Nakarai Chemical Co., Tokyo, Japan). The remaining blood, which was taken for ghrelin assay, was treated as described earlier.

\section{RIA}

After the plasma had been diluted with an equal volume of $0.9 \%$ saline, it was applied to a Sep-Pak C-18 cartridge that had been pre-equilibrated with $0.9 \%$ saline. The cartridge was washed with saline and $10 \% \mathrm{CH}_{3} \mathrm{CN}$ solution containing $0 \cdot 1 \%$ trifluoroacetic acid (TFA). The absorbed peptides were eluted with $60 \% \mathrm{CH}_{3} \mathrm{CN}$ solution containing $0 \cdot 1 \%$ TFA, lyophilized, then subjected to RIA. Two polyclonal antibodies, one against the N-terminal fragment of ghrelin (n-octanoylated rat [1-11] ghrelin) and the other against the C-terminal fragment of ghrelin (rat [13-28] ghrelin), were produced in a rabbit. Details of the 
method of preparation and characterization of this antibody have been described elsewhere (Kojima et al. 1999). The anti-rat ghrelin [1-11] antiserum \#G606 specifically recognized ghrelin with an $n$-octanoylated Ser 3 and did not recognize des-acyl ghrelin. Synthetic rat $\left[\mathrm{Tyr}^{29}\right]-$ ghrelin[1-28] was radioiodinated by the lactoperoxidase method. The ${ }^{125}$ I-labeled peptide was then purified by RP-HPLC on TSK ODS SIL 120A column (Tosoh Co. Ltd, Tokyo, Japan). The RIA incubation buffer was $50 \mathrm{mM}$ sodium phosphate $(\mathrm{pH} 7 \cdot 4$ ) containing $0 \cdot 5 \%$ bovine serum albumin (BSA; treated with $\mathrm{N}$ ethylmaleimide), $80 \mathrm{mM} \mathrm{NaCl}, 25 \mathrm{mM}$ EDTA-2Na, $0.05 \% \mathrm{NaN}_{3}$ and $0.5 \%$ Triton $\mathrm{X}-100$. For the assay, a diluted sample or standard solution $(100 \mu \mathrm{l})$ was incubated for $24 \mathrm{~h}$ with $100 \mu \mathrm{l}$ anti-ghrelin antiserum (final dilution 1/620 000). The tracer solution (16 000 c.p.m. in $100 \mu \mathrm{l}$ ) was added, and the mixture was incubated for a further $24 \mathrm{~h}$. The bound and free ligands were separated by a second antibody $(200 \mu \mathrm{l})$. All procedures were carried out at $4{ }^{\circ} \mathrm{C}$, and all plasma samples were assayed in duplicate. The half-maximum inhibition level produced by rat ghrelin [1-28] on the standard RIA curve was $3 \cdot 8 \mathrm{fmol} /$ tube with anti-ghrelin [1-11] antiserum (\#606) and 80 fmol with anti-ghrelin [13-28] antiserum (\#G107). The intra- and inter-assay coefficients of variation of the RIA for the ghrelin N-terminal fragment (\#G606) at 50\% binding were $3 \cdot 5 \%$ and $3 \cdot 2 \%$ respectively, while those of RIA for the ghrelin C-terminal fragment (\#G107) were $3 \cdot 7 \%$ and $3 \cdot 3 \%$ respectively. The mean ( \pm S.E.M.) recoveries of rat ghrelin [1-28] (1 ng) and ${ }^{125} \mathrm{I}$-rat ghrelin [1-28] (5000 c.p.m.), which were added to the rat plasma then extracted using Sep-Pak C-18 cartridges (Waters, Milford, MA, USA), were $92 \cdot 2 \pm 0 \cdot 4 \%$ and $88 \cdot 9 \pm 0 \cdot 6 \%$ respectively.

The statistical analysis was carried out by analysis of variance with the post hoc Fisher test. $P<0.05$ was considered significant.

\section{Results}

Daily i.c.v. injections of $0 \cdot 1$ and $1 \mu \mathrm{g}$ anti-ghrelin $\operatorname{IgG}$ gradually decreased both daily food intake and body weight in a dose-dependent manner (Fig. 1). The average of daily food intake was significantly reduced by $1 \mu \mathrm{g}$ anti-ghrelin $\operatorname{IgG}$. The average body weight gain per day was significantly reduced by both $0 \cdot 1 \mu \mathrm{g}$ and $1 \mu \mathrm{g}$ antighrelin IgG. The effect of over $2 \mu \mathrm{g}$ of IgG could not be estimated because the same level of pre-immune IgG also decreased food intake and body weight gain. Since levels of acyl ghrelin and total ghrelin were measured by RIA using the anti-ghrelin antiserum against the $\mathrm{N}$-terminal region (rat [1-11] ghrelin) and that against the C-terminal region (rat [13-28] ghrelin) respectively, expected desacyl ghrelin levels may be calculated using the following equation: des-acyl ghrelin $=$ total ghrelin - acyl ghrelin .
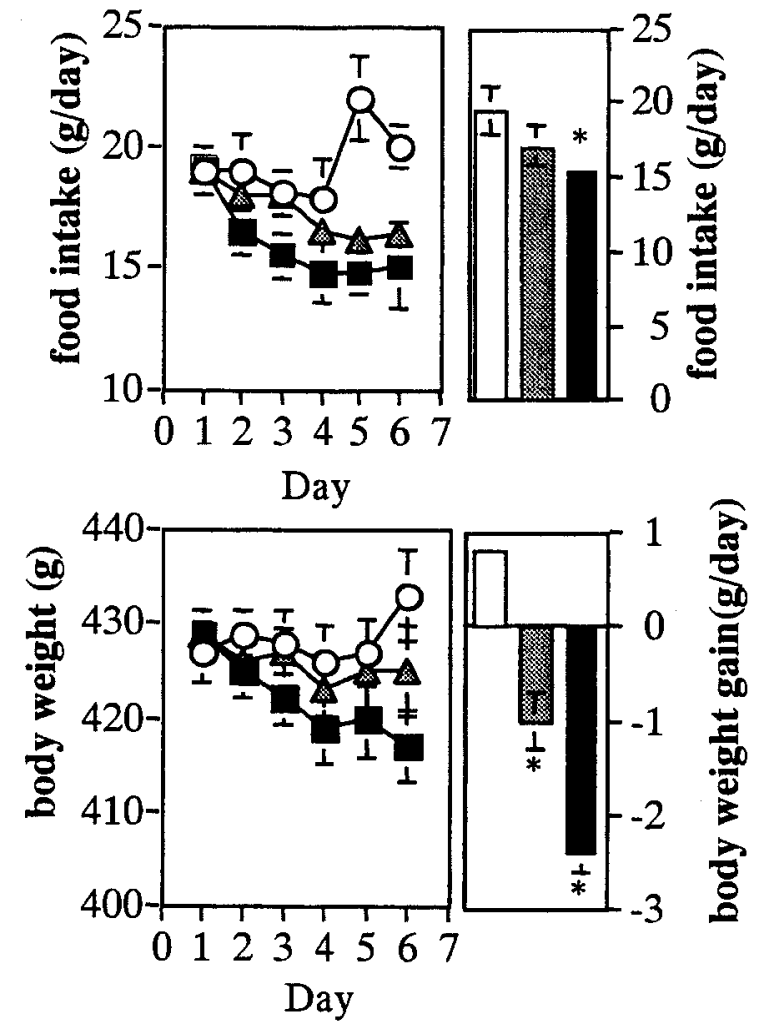

Figure 1 Effect of daily i.c.v. treatment with anti-ghrelin IgG on daily food intake and body weight gain: $1 \mu \mathrm{g} / 10 \mu \mathrm{l}$ pre-immune IgG (open circle and open bar), $0 \cdot 1 \mu \mathrm{g} / 10 \mu \mathrm{l}$ (gray triangle and gray bar) or $1 \mu \mathrm{g} / 10 \mu \mathrm{l}$ (black square and black bar) anti-ghrelin IgG raised against the $\mathrm{N}$-terminal region of ghrelin were injected twice a day (at $0845 \mathrm{~h}$ and $1845 \mathrm{~h}$ ) for 5 days. The left panels represent the daily change of food intake and body weight. The right panels represent the average food intake and body weight gain per day. The symbols and bars represent mean values, the vertical lines are \pm S.E.M. $(n=8)$, and the asterisks indicate significant differences $(P<0 \cdot 05$ vs pre-immune $\lg$ alone).

Both acyl ghrelin and total ghrelin levels in the gastric vein were higher than those in the trunk (Fig. 2). Total ghrelin was higher by over fivefold compared with acyl ghrelin in the gastric vein and trunk. However, there was no significant difference in calculated des-acyl ghrelin between the gastric vein and trunk (gastric vein: $0.62 \pm 0.08 \mathrm{pmol} / \mathrm{ml}$ vs trunk: $0.51 \pm 0.06 \mathrm{pmol} / \mathrm{ml}$ ). The $12 \mathrm{~h}$ of fasting of dark-phase feeding significantly increased both acyl and total ghrelin levels in the gastric vein. Similarly, a single i.p. administration of CCK or gastrin increased significantly both acylated and total ghrelin levels in the gastric vein (Fig. 3). The expected des-acyl ghrelin levels were as follows: saline $1 \cdot 15 \pm 0 \cdot 16 \mathrm{pmol} / \mathrm{ml}$, fasting $1 \cdot 57 \pm 0 \cdot 24 \mathrm{pmol} / \mathrm{ml}$, gastrin $1.89 \pm 0.25 \mathrm{pmol} / \mathrm{ml}(P<0.05$ vs saline $)$, and CCK $3 \cdot 38 \pm 0.042 \mathrm{pmol} / \mathrm{ml}(P<0 \cdot 05$ vs saline $)$. However, electrical stimulation of the left cervical vagus nerve did not 


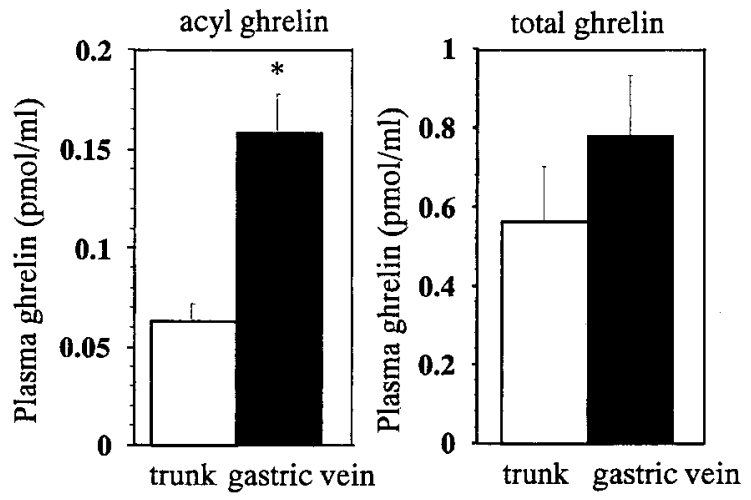

Figure 2 Comparison of plasma acyl and total ghrelin levels between the gastric vein and trunk. Acyl ghrelin and total ghrelin were measured by RIA using the anti-ghrelin antiserum raised against $\mathrm{N}$-terminal ( $n$-octanoylated, rat [1-11] ghrelin) and that raised against the C-terminal (rat [13-28] ghrelin) region respectively. The bars and vertical lines represent mean values \pm S.E.M. $(n=8)$. ${ }^{*} P<0 \cdot 05$ vs trunk.
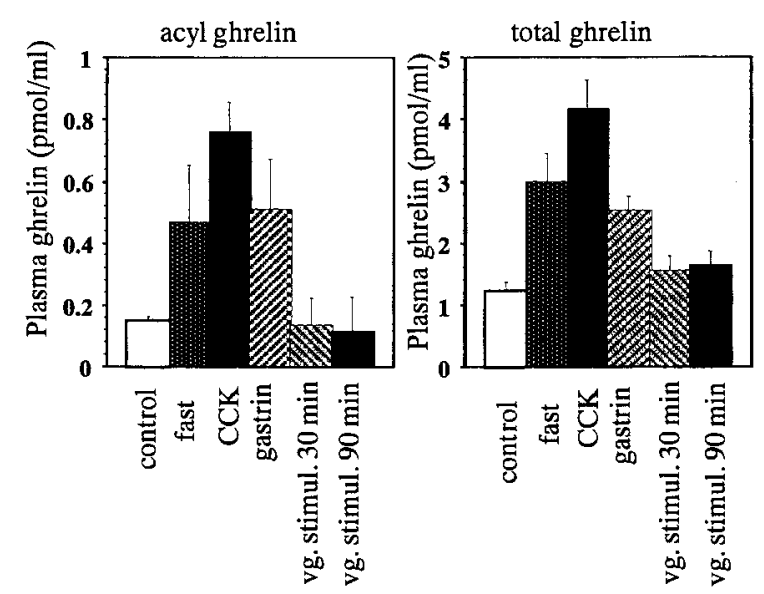

Figure 3 Effect of $12 \mathrm{~h}$ of fasting, an i.p. injection of gastrin or CCK, and electrical stimulation of the vagus nerve on plasma ghrelin levels. Blood samples were collected from the gastric vein and from the heart $12 \mathrm{~h}(1900-0700 \mathrm{~h})$ after fasting, and $40 \mathrm{~min}$ after an i.p. injection of CCK ( $20 \mu \mathrm{g} / \mathrm{kg}$ body weight), gastrin ( $2 \mu \mathrm{g} / \mathrm{kg}$ body weight) or saline, and 30 or $90 \mathrm{~min}$ after electrical stimulation of the left cervical vagus nerve (vg. stimul.). The bars and vertical lines represent mean values \pm S.E.M. $(n=8)$.

influence either the acyl or total ghrelin levels in the gastric vein (Fig. 3). Both the acyl and total ghrelin levels exhibited a diurnal pattern of release, with bimodal peaks occurring in free-feeding rats. The first and second peaks were observed at $1500 \mathrm{~h}$ into the light period and at $0600 \mathrm{~h}$ into the dark period respectively (Fig. 4). Although blood glucose levels were almost constant during the day, the volume of gastric contents exhibited a diurnal rhythm, with the increase and decrease occurring during the dark and light period respectively.
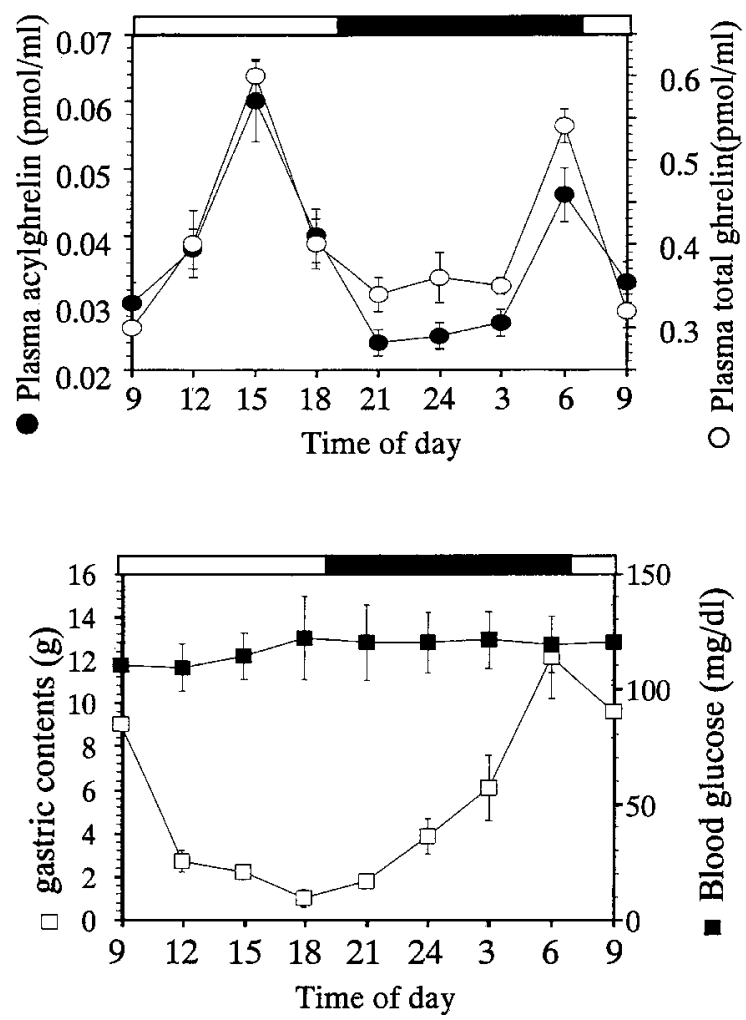

Figure 4 Diurnal pattern of plasma ghrelin levels, blood glucose levels and volume of gastric contents. The blood was collected by decapitation. The open and closed circles represent the total ghrelin and acyl levels respectively. Please note the scale difference. The open and closed squares represent the volume of gastric contents and blood glucose respectively. The symbols and vertical lines represent mean values \pm S.E.M. $(n=8)$. The upper white and dark bars indicate the light and dark periods respectively.

\section{Discussion}

It has been discovered recently that ghrelin is an endogenous ligand for GHS-R in rat and human stomachs (Kojima et al. 1999). Immunohistochemical studies have revealed that in addition to the stomach, ghrelin may be found in the hypothalamic arcuate nucleus (Kojima et al. 1999). Accumulating data on the physiological role of ghrelin indicate that it may play an important role in the secretion of GH (Kojima et al. 1999, Date et al. 2000b, Wren et al. 2000), gastric acid (Date et al. 2001), insulin, gastrin (Lee et al. 2002) and luteinizing hormone (Furuta et al. 2001), and in the control of food intake (Tschop et al. 2000, Asakawa et al. 2001, Nakazato et al. 2001). In the present study, daily i.c.v. administrations of anti-ghrelin antiserum to neutralize the centrally released ghrelin decreased significantly daily food intake and body weight gain, supporting the hypothesis that endogenous centrally released ghrelin may be involved in the control of daily food intake. However, contrary to our expectation, the 
suppressing effect of daily food intake by anti-ghrelin antiserum was small, since the i.c.v. injection of even small doses $(10 \mathrm{pmol})$ of ghrelin was shown to significantly stimulate food intake (Nakazato et al. 2001). Since, as we observed in a previous report, a single injection of $0.5 \mu \mathrm{g}$ of anti-ghrelin IgG suppressed food intake to half or less in 8-h fasted rats, it is unlikely that the low level of suppression effected by anti-ghrelin antiserum is the result of insufficient anti-ghrelin. Therefore, the participation of centrally released ghrelin in controlling daily food intake may not be physiologically predominant. On the other hand, it has been reported recently that in mice, vagotomy inhibits the food intake induced by peripheral administration of ghrelin, suggesting that endogenous peripherally released ghrelin stimulates food intake via a mainly peripheral action (Lee et al. 2002). Further study is required to elucidate the mechanism of action of ghrelin in the control of food intake.

From their morphology and distribution, the cells in the stomach that were immunostained with the anti-serum against the $\mathrm{N}$-terminal region of the peptide were considered to be X/A-like cells whose hormonal product was previously unclear (Date et al. 2000). Analysis by RPHPLC has revealed that both acyl and des-acyl ghrelin exist in the rat gastric fundus at a ratio of 1·3:1 (des-acyl ghrelin : acyl ghrelin) (Toshinai et al. 2001). These results indicate the possibility that both acyl and des-acyl ghrelin are secreted from X/A-like cells. In the present study, plasma ghrelin levels were measured using two kinds of anti-ghrelin antiserum. The antiserum raised against the $\mathrm{N}$-terminal region of ghrelin recognized acylated ghrelin only, which seems to be actively involved in GH secretion. The antiserum raised against the C-terminal region of ghrelin recognized both acylated and des-acylated ghrelin (total ghrelin). The fact that des-acyl ghrelin levels were about fourfold higher than acyl ghrelin suggests that the des-acyl form of ghrelin is dominant in the blood. In a comparison between gastric vein and trunk levels, however, levels of acyl ghrelin were higher in the gastric vein than in the trunk, indicating that acyl ghrelin is the predominantly secreted form in the stomach. Furthermore, these results suggest that endogenous ghrelin is easily and rapidly decomposed after secretion from the stomach, or that the decomposed fragments are retained in the blood for a long period of time. These observations were consistent with the preliminary results obtained by analysis by RP-HPLC (data not shown). The 12-h fast stimulated acyl ghrelin secretion from the stomach. This result is partially consistent with previous reports showing that 48 or $72 \mathrm{~h}$ of fasting increases plasma ghrelin levels, and re-feeding decreases these levels (Tschop et al. 2000, Toshinai et al. 2001, Lee et al. 2002). However, this study indicates that even half a day of emptiness can stimulate ghrelin secretion from the stomach. Under empty conditions, vagus nerve activity is reduced. In the present study, electrical stimulation of the vagus nerve did not increase acyl ghrelin secretion, rather it showed a tendency to decrease. Stimulation of the vagus nerve is thought to have similar effects to high activity of the vagus nerve under fully fed conditions. Therefore, these results may indicate that activity of the vagus nerve suppresses ghrelin secretion. This speculation is supported by the fact that vagotomy increases plasma ghrelin levels (Lee et al. 2002).

The i.p. administration of gastrin and CCK increased ghrelin secretion from the stomach. Both acyl and des-acyl ghrelin levels were increased in the gastric vein within 40 min of administration, suggesting that gastrin and CCK rapidly stimulate ghrelin secretion. However, the interpretation of these results is difficult, since gastrin and CCK are usually secreted after feeding and, conversely, their levels are reduced by fasting. In addition, CCK increases vagus nerve activity. Therefore, it should be assumed that, in general, gastrin and CCK reduce ghrelin secretion. On the other hand, ghrelin has been shown to stimulate gastric acid secretion via stimulation of the vagus nerve. Therefore, gastrin and CCK may stimulate contemporary ghrelin to stimulate gastric acid secretion.

We observed diurnal change in plasma ghrelin levels in free-feeding rats. Both acyl and des-acyl ghrelin express the same pattern with two peaks, one at $1500 \mathrm{~h}$ into the light period and one at $0600 \mathrm{~h}$ into the dark period. Although the physiological significance of these peaks is unknown, there was no correlation with blood glucose levels. On the other hand, the first peak of ghrelin at $1500 \mathrm{~h}$ into the light period and the second peak at $0600 \mathrm{~h}$ into the dark period are consistent with the lowest volume and greatest volume of gastric contents respectively. This indicates that ghrelin secretion is increased under conditions of both gastric emptying and filling. Therefore, one possible explanation is that the function of the peak in ghrelin levels at $1500 \mathrm{~h}$ into the light period is to stimulate food intake, and that the peak at $0600 \mathrm{~h}$ into the dark period is to stimulate gastric acid secretion. If this is the case, gastrin and CCK may participate in the later peak.

In conclusion, although ghrelin plays an important role in the control of food intake, the site of action may be different between centrally and peripherally released ghrelin. Acyl ghrelin is secreted from the stomach into the gastric vein; the regulation of this secretion seems to be complex, because peripheral ghrelin levels are highest under both gastric emptying and filling conditions. These bimodal peaks, which occur under contradictory conditions, may each play different physiological roles. Further studies are required to elucidate the regulatory mechanism underlying ghrelin secretion, and its physiological roles.

\section{Acknowledgements}

This research was supported by a grant-in-aid for Scientific Research from the Japan Society for the promotion of Science. 


\section{References}

Asakawa A, Inui A, Kaga T, Yuzuriha H, Nagata T, Ueno N, Makino S, Fujimiya M, Niijima A, Fujino MA \& Kasuga M 2001 Ghrelin is an appetite-stimulatory signal from stomach with structural resemblance to motilin. Gastroenterology 120 337-345.

Date Y, Kojima M, Hosoda H, Sawaguchi A, Mondal MS, Suganuma T, Matsukura S, Kangawa K \& Nakazato M 2000a Ghrelin, a novel growth hormone-releasing acylated peptide, is synthesized in distinct endocrine cell type in the gastrointestinal tracts of rats and humans. Endocrinology 141 4255-4261.

Date Y, Murakami N, Kojima M, Kuroiwa T, Matsukura S, Kangawa K \& Nakazato M 2000 b Central effects of a novel acylated peptide, ghrelin, on growth hormone release in rats. Biochemical and Biophysical Research Communications 275 477-480.

Date Y, Nakazato M, Murakami N, Kojima M, Kangawa K \& Matsukura S 2001 Ghrelin acts in the central nervous system to stimulate gastric acid secretion. Biochemical and Biophysical Research Communications 280 904-907.

Furuta M, Funabashi T \& Kimura F 2001 Intracerebroventricular administration of ghrelin rapidly suppresses pulsatile luteinizing hormone secretion in ovariectomized rats. Biochemical and Biophysical Research Communications 288 780-785.

Hosoda H, Kojima M, Matsuo H \& Kangawa K 2000 Purification and characterization of rat des-Gln14-Ghrelin, a second endogenous ligand for the growth hormone secretagogue receptor. Journal of Biological Chemistry 275 21995-22000.

Kamegai J, Tamura H, Shimizu T, Ishii S, Sugihara H \& Wakabayashi I 2001 Chronic central infusion of ghrelin increases hypothalamic neuropeptide $\mathrm{Y}$ and Agouti-related protein mRNA levels and body weight in rats. Diabetes 50 2438-2443.
Kojima M, Hosoda H, Date Y, Nakazato M, Matsuo H \& Kangawa K 1999 Ghrelin is a growth-hormone-releasing acylated peptide from stomach. Nature 402 656-660.

Lee H-M, Wang G, Englander EW, Kojima M \& Greeley GH 2002 Ghrelin, a new gastrointestinal endocrine peptide that stimulates insulin secretion: enteric distribution, ontogeny, influence of endocrine, and dietary manipulations. Endocrinology 143 185-190.

Nakazato M, Murakami N, Date Y, Kojima M, Matsuo H, Kangawa K \& Matsukura S 2001 A role for ghrelin in the central regulation of feeding. Nature 409 194-198.

Shintani M, Ogawa Y, Ebihara K, Aizawa-Abe M, Miyanaga F, Takaya K, Hayashi T, Inoue G, Hosoda H, Kojima M, Kanagawa K \& Nakao K 2001 Ghrelin, an endogenous growth hormone secretagogue, is a novel orexigenic peptide that antagonizes leptin action through the activation of hypothalamic neuropeptide Y/Y1 receptor pathway. Diabetes $\mathbf{5 0} 227-232$.

Toshinai K, Mondal MS, Nakazato M, Date Y, Murakami N, Kojima M, Kanagawa K \& Matsukura S 2001 Upregulation of Ghrelin expression in the stomach upon fasting, insulin-induced hypoglycemia, and leptin administration. Biochemical and Biophysical Research Communications 281 1220-1225.

Tschop M, Smiley DL \& Heiman ML 2000 Ghrelin induces adiposity in rodents. Nature 407 908-913.

Wren AM, Small CJ, Ward HL, Murphy KG, Dakin CL, Taheri S, Kennedy AR, Roberts GH, Morgan DG, Ghatei MA \& Bloom SR 2000 The novel hypothalamic peptide ghrelin stimulates food intake and growth hormone secretion. Endocrinology 141 4325-4328.

Received 18 April 2002

Accepted 29 April 2002 